\title{
Relationship between thrust, anthropometrics, and dry-land strength in a national junior swimming team
}

Jorge E Morais, Mário C Marques, David Rodríguez-Rosell, Tiago M Barbosa \& Daniel A Marinho

To cite this article: Jorge E Morais, Mário C Marques, David Rodríguez-Rosell, Tiago M Barbosa \& Daniel A Marinho (2019): Relationship between thrust, anthropometrics, and dryland strength in a national junior swimming team, The Physician and Sportsmedicine, DOI: 10.1080/00913847.2019.1693240

To link to this article: https://doi.org/10.1080/00913847.2019.1693240

Accepted author version posted online: 15

Nov 2019.

Published online: 30 Nov 2019.

Submit your article to this journal ¿

Џ Article views: 7

View related articles

View Crossmark data $₫$ 


\title{
Relationship between thrust, anthropometrics, and dry-land strength in a national junior swimming team
}

\author{
Jorge E Morais (1) ${ }^{\mathrm{a}, \mathrm{b}, \mathrm{d}}$, Mário C Marques (1) ${ }^{\mathrm{a}, \mathrm{d}}$, David Rodríguez-Roselle, Tiago M Barbosa (10) ${ }^{\mathrm{b}, \mathrm{c}, \mathrm{d}}$ and Daniel A Marinho
}

aDepartment of Sport Sciences, University of Beira Interior, Covilhã, Portugal; 'bepartment of Sport Sciences, Instituto Politécnico de Bragança, Bragança, Portugal; 'Nanyang Technological University, National Institute of Education, Singapore; dResearch Centre in Sports, Health and Human

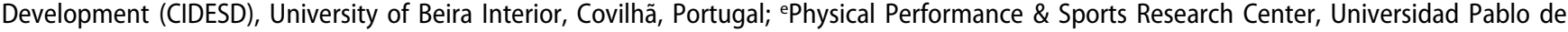
Olavide, Seville, Spain

\begin{abstract}
Objectives: This study aimed to (i) assess an anthropometric and thrust inter-limb asymmetry, and; (ii) determine the contribution of anthropometrics, and dry-land upper-body strength and power to the thrust of talented adolescent swimmers.

Methods: Eighteen talented adolescent swimmers ( 12 boys and 6 girls: $15.81 \pm 1.62$ years old) were evaluated. A set of anthropometric, dry-land upper-body strength and power, and in-water thrust were assessed.

Results: Despite the fact that the dominant side presented higher values in anthropometrics (except for the hand surface area) and thrust, non-significant inter-limb differences were found. The symmetry index indicated a symmetry between upper-limbs. Hierarchical linear modeling retained as main predictors of each upper-limb thrust the respective hand surface area (dominant upper limb: estimate $=0.293,95 \mathrm{Cl}$ : $0.117 ; 0.469, p=0.005$; non-dominant upper limb: estimate $=0.295,95 \mathrm{Cl}: 0.063 ; 0.526, p=0.025)$. The full stroke cycle retained the upper-body dry-land strength as main predictor (estimate $=0.397,95 \mathrm{Cl}: 0.189$; $0.605, \mathrm{p}=0.002$ ).

Conclusion: The hand surface area and upper-body strength were the main predictors of each upperlimb and full stroke cycle thrust, respectively. Hence, coaches and practitioners should aim to carefully maximize the hand surface area (by finger spreading) while performing the stroke, as well as dry-land upper-body strength in order to enhance the performance.
\end{abstract}

\section{ARTICLE HISTORY}

Received 23 July 2019

Accepted 11 November 2019

\section{KEYWORDS}

Swimming; strength; body dimensions; in-water force

\section{Introduction}

Swimmers should minimize drag and increase thrust to enhance the swim speed [1]. During the clean swimming phase, swimmers are submitted to water resistance, i.e. active drag [1]. In this context, although the drag effect (namely active) on swimmers are vastly reported in the literature [2], less is known about the thrust. Indeed, the assessment of the swimmer's propulsive force (i.e. thrust) is still difficult to quantify, and hence an interesting topic among researchers and practitioners $[3,4]$.

Video-recording methods were used to measure the swimmer's hand and/or arm movements based on threedimensional reconstructions $[5,6]$. However, it was noted that the thrust forces were not measured directly but estimated, this being a major limitation of the kinematic method [3]. Others used tethered swimming as a method to measure the maximum force (i.e. thrust) [7]. It is suggested that when swimming at a constant velocity, the propulsive forces produced by the swimmer should equal the resistive ones [8]. On the other hand, there is no evidence that the force produced is equal when swimming 'freely' and tethered [9]. Conversely, the pressure on propelling surfaces over each stroke can be measured by sensors placed at strategic positions on the hand's surface, and hence measured directly $[10,11]$. In the front crawl, the thrust force is primarily created by the swimmer's upper-limbs as they move through the water [12]. The swimmers' hands might be seen as the key body part responsible for the major source of the upper limbs' thrust, since their trajectory and orientation are responsible for controlling the thrust generated by the upper-limbs [13]. Thus, it is reasonable to think that it is an important performance area for optimizing swimming thrust, which could present a positive effect on swim velocity $[14,15]$.

Indeed, it was highlighted that the upper-limbs are responsible for about $90 \%$ of the total propulsion in the front crawl [16]. Zamparo et al. [17] showed that propelling efficiency plays a key-role in swimmer's thrust, and the first one is highly dependent on anthropometrics (namely the upper-limbs). Therefore, it can be suggested that upper limbs' anthropometrics also contribute to front crawl thrust. Moreover, it was suggested that the use of only the hand and forearm to estimate a swimmer's thrust might lead to underestimated values, enhancing the importance of including the upperarm as well [18]. There is not a solid body of knowledge about inter-limb asymmetries in anthropometric features and 
upper-limbs' thrust, or of their relationship [19]. In connection with the above, it was pointed out that post-pubertal male swimmers presented an asymmetrical force exertion, where dominant upper-limbs showed higher force values in comparison to the non-dominant ones $[20,21]$. However, to the best of our knowledge, there is no solid evidence about how anthropometrics (specifically the upper-limbs as an articulated segment, i.e. several segments) contribute to/influence swimming thrust.

Additionally, strength and power are required to produce in-water thrust [8]. Dry-land strength training programs seem to have a positive effect on adolescent swimmers' performance. That is, higher values of dry-land strength and power enable swimmers to produce higher in-water thrust, and consequently higher swim velocity $[22,23]$. Indeed, a moderate/ high association between dry-land variables and the swimmer stroke mechanics has been verified [8]. Moreover, it was shown that concurrent dry-land and in-water programs were more efficient than the swimming program alone in increasing performance in adolescent sprint swimmers [24]. Thus, results of these studies suggest that developing strength and power on land led to an enhancement of swimmers' performance [24], and this could occur due to the in-water thrust improvement [23]. Nonetheless, there is no clear evidence about how dry-land strength and power may contribute specifically to swimmers' in-water thrust. It has been suggested that the upper-body strength and power needed to displace the swimmer at front crawl is based on a set of several upper-body muscles [25]. Thus, one might claim that a higher upper-body strength and power would have a positive and direct effect on swimmers' in-water thrust. Moreover, hypothetical interactions between anthropometric features and dry-land strength and power may be highly representative of front crawl in-water thrust.

Therefore, based on the above considerations, the main aims of this study were to (i) assess a hypothetical anthropometric and thrust inter-limb asymmetry, and; (ii) determine the contribution of anthropometrics, and dry-land upper-body strength and power to the thrust of talented adolescent swimmers. It was hypothesized that a significant inter-limb effect would be verified (for both anthropometrics and thrust). Moreover, some anthropometric features, and/or upper-body strength and power, or interactions between such variables, would have a positive and significant effect on front crawl thrust.

\section{Materials and methods}

\section{Participants}

Eighteen talented adolescent swimmers ( 12 boys and 6 girls: $15.81 \pm 1.62$ years old; boys' FINA points: $572.17 \pm 67.32$; girls' FINA points: $636.33 \pm 105.60$ at short course $100 \mathrm{~m}$ freestyle) were recruited and evaluated. These swimmers were part of a national junior team, including swimmers participating in international championships, national record holders, and agegroup national champions. They had more than 5 years of competitive experience and trained six to seven swimming sessions per week, and two to $3 \mathrm{~h}$ of dry-land strength and power training per week under the direction of the same coach. Parents or guardians, and the swimmers themselves signed an informed consent form. All procedures were in accordance to the Declaration of Helsinki regarding human research, and the University Ethics Board approved the research design.

\section{Experimental protocol}

Swimmers were recruited to analyze the relationship between anthropometric features and upper-body strength with swimming thrust (cross-sectional study). At the assessment time, they were in peak-performance (end of the second macrocycle). To ensure a proper reproduction of the experimental trials, all swimmers underwent a familiarization process as part of their in-water and strength and conditioning testing. Moreover, they were asked about their upper-limb dominance (self-report) for data analysis. The in-water and strength and conditioning tests were performed on different days $(72 \mathrm{~h})$ to ensure that one test would not affect the other. The in-water experimental testing took place ina $25 \mathrm{~m}$ indoor swimming pool (water temperature: $27.5^{\circ} \mathrm{C}$; air temperature: $26.0^{\circ} \mathrm{C}$; relative humidity: $66 \%$ ). Before the in-water tests, swimmers performed a $1000 \mathrm{~m}$ standard warm-up. The dry-land strength test was conducted in a gym by a certified strength and conditioning coach who was responsible for the entire team dry-land training. Two trials were performed (on separate but consecutive days) to ensure full recovery. Only the best repetition was considered for analysis as reported elsewhere [26].

\section{Anthropometry}

The height $(\mathrm{H})$ was measured with a digital stadiometer (SECA, 242, Hamburg, Germany). The body mass (BM) was measured with a digital scale (TANITA, BC-730, Amsterdam, Netherlands). The arm span (AS), upper-limb length, and the hand's surface area (HSA) were measured by digital photogrammetry [27]. For the AS, swimmers were photographed near a $2 \mathrm{D}$ calibration frame, in an orthostatic position, with both arms in lateral abduction at a $90^{\circ}$ angle to the trunk. Both arms and fingers were fully extended. The distance between the tip of each third finger was measured. The upper-limbs' length was measured as an articulated segment. Light markers were placed on each upper-limb acromion, lateral epicondyle, and styloid process. Afterward, the arm was measured between the acromion and the lateral epicondyle, and the forearm between the lateral epicondyle and the styloid process. For the HSA, swimmers placed their hand (one at each time) on the scan surface of a copy machine, and the file was exported to a PC. The scan surface was also fitted with a $2 \mathrm{D}$ calibration frame. The distances and surface areas were measured with a dedicated software [27].

\section{Dry-land strength}

The bench press test (Multipower Fitness Line, Peroga, Spain) was used to evaluate the overall upper-body strength on dryland. This is described in the literature as the most frequently used test to assess upper-body strength [28], since it recruits 
several muscles related to front crawl stroke $[8,29]$. Moreover, in swimming, the arm's thrust is based on the ability to generate force recruiting a large set of muscles. Therefore, the bench press test seems to be the most appropriate test to evaluate all those muscles in one single test [22]. Before the test, swimmers performed bench press warm-up sets to get familiarized with the machine. The maximum upper-body strength (1RM) was estimated based on the bar velocity test [26]. To measure the bar velocity, a dynamic measurement system (T-Force Measurement System, Ergotech, Murcia, Spain) was attached to the bar. This consists of a cableextension linear velocity transducer interfaced to a personal computer, which automatically calculates the relevant kinematic and kinetic variables of every repetition [26]. Each swimmer was instructed to lower the bar to the chest, and wait there until hearing a start command. This momentary pause is used to minimize the contribution of the rebound effect and allow more reproducible and consistent assessments [26,30]. The swimmers were carefully instructed to always perform each trial at maximal intended velocity. The test was monitored by an experienced and certified strength and conditioning evaluator. Initial load was set at $20 \mathrm{~kg}$ for all swimmers, and was progressively increased in $10 \mathrm{~kg}$ increments until the attained mean propulsive velocity was lower than $0.5 \mathrm{~m} \cdot \mathrm{s}^{-1}$. Thereafter, the load was adjusted in smaller increments (1 to $5 \mathrm{~kg}$ ), individually for each swimmer, determining the 1RM with higher precision. The heaviest load that each swimmer could properly lift to the full extension of their elbows was considered to be their1RM [30]. Swimmers performed three attempts with the lighter loads (mean propulsive velocity $\left.>1.0 \mathrm{~m} \cdot \mathrm{s}^{-1}\right)$, two with the medium $(0.65 \leq$ mean propulsive velocity $\leq 1.0 \mathrm{~m} \cdot \mathrm{s}^{-1}$ ), and one with the heaviest load (mean propulsive velocity $<0.65 \mathrm{~m} \cdot \mathrm{s}^{-1}$ ). They rested $3 \mathrm{~min}$ for the lighter and medium loads, and $6 \mathrm{~min}$ for the heaviest one. This protocol was reproduced as reported elsewhere [26].

\section{Thrust}

Swimmers were invited to perform three maximal all-out trials of $25 \mathrm{~m}$ in the front crawl with a push-off start. A force data acquisition equipment Aquanex + Video (Swimming Technology Research, USA) was used to measure thrust ( $f=$ $100 \mathrm{~Hz}$ ). This system is based on sensors that estimate in-water force, with a measurement error of $0.2 \%$ [11]. The validation and reliability processes were previously presented in other studies [11]. Such sensors were placed between the third and fourth metacarpals to measure the pressure differential between the palmar and dorsal surfaces. At the beginning of each trial, swimmers were asked to keep their hands immersed at the waistline for $10 \mathrm{~s}$ in order to calibrate the system with the hydrostatic pressure values. The video camera was placed at the side of the swimming pool (recording the swimmers on the sagittal plane) (Figure 1 - Panel C). The sensors and video output were connected to an A/D converter connected to a laptop on the pool deck with the Aquanex software (Aquanex v. 4.2 C1211, Richmond, USA) [10]. Afterward, time-force series were imported into a signal processing software (AcqKnowledge v. 3.9.0, Biopac Systems, Santa Barbara, USA). The signal was handled with the Butterworth fourth-order low-pass filter (cutoff: $5 \mathrm{~Hz}$ ). The mean value of each thrust variable was analyzed based on three consecutive stroke cycles between the 11th and 24th mark (ICC = 0.98). Afterward, the mean of the three trials was used for analysis.

For each dominant and non-dominant arm-pull (i.e. underwater phase), the mean propulsive force (F_mean_dominant and F_mean_non-dominant, $N$ ), and the peak force (F_peak_dominant and F_peak_non-dominant, N) were assessed. Afterward, the F_stroke cycle (the total force produced in one full stroke cycle, N) and F_peak_stroke cycle (the peak force produced in one full stroke cycle, $N$ ) were computed. The intra-cyclic force variation of each upper-limb ( $\mathrm{dF}$ _dominantand $\mathrm{dF}$ _non-dominant, \%), and of the full stroke cycle (dF_stroke cycle, \%) was calculated as follows [31]:

$$
d F=\frac{\sqrt{\frac{\sum_{i}\left(\text { Force }_{i}-\overline{\text { Force }}\right) \cdot F_{i}}{n}}}{\frac{\sum_{i} \text { Force }_{i} \cdot F_{i}}{n}} \cdot 100
$$

where $d F$ is the intra-cyclic variation of the force (\%), Force is the mean force $(N)$, Force $e_{i}$ is the instant force $(N), F_{i}$ is the acquisition frequency, and $\mathrm{n}$ is the number of observations. The symmetry index (SI) was computed as [32]:

$$
S I=\frac{x_{d}-x_{n d}}{0.5\left(x_{d}+x_{n d}\right)} \cdot 100
$$

where $x_{d}$ and $x_{n d}$ are the mean values for the dominant and non-dominant upper-limbs' variables, respectively. The authors deemed the symmetry index within these cutoff values: $-10 \%<\mathrm{SI}<10 \%$ indicates symmetry, and $\mathrm{SI}<-10 \%$ and $\mathrm{SI}>10 \%$ indicates asymmetry [32].

\section{Statistical analyses}

Correlation agreements (thrust variables versus anthropometric and upper-body dry-land strength) between both sexes were computed with the Fischer's z-score [33]. Non-significant differences were verified between correlations ( $p>0.05$ ), suggesting that both sexes could be pooled together. The Shapiro-Wilk and the Levene tests were used to assess the normality and homoscedasticity, respectively. The mean plus one standard deviation, $95 \%$ confidence interval $(95 \mathrm{Cl})$, minimum, and maximum were computed as descriptive statistics. One-way ANOVA $(p<0.05)$ was computed to verify a side effect (i.e. variation between the dominant and non-dominant limbs). Cohen's d was selected as standardized effect size, and deemed as: (i) small effect size $0 \leq 1$ $\mathrm{d} \mid \leq 0.2$; (ii) medium effect size if $0.2<|\mathrm{d}| \leq 0.5$ and; (iii) large effect size if $|d|>0.5$ [34]. Hierarchical linear modeling (HLM) was used to verify the thrust predictors for the full stroke cycle, dominant arm-pull, and non-dominant arm-pull. For each thrust variable, all independent variables were input, and the final models only retained significant predictors. The maximum likelihood estimation was computed by HLM7 software [35].

\section{Results}

Table 1 presents the descriptive statistics for all the variables assessed. Table 2 reports the results for the inter-limb effect (i.e. dominant vs non-dominant). There was a non- 

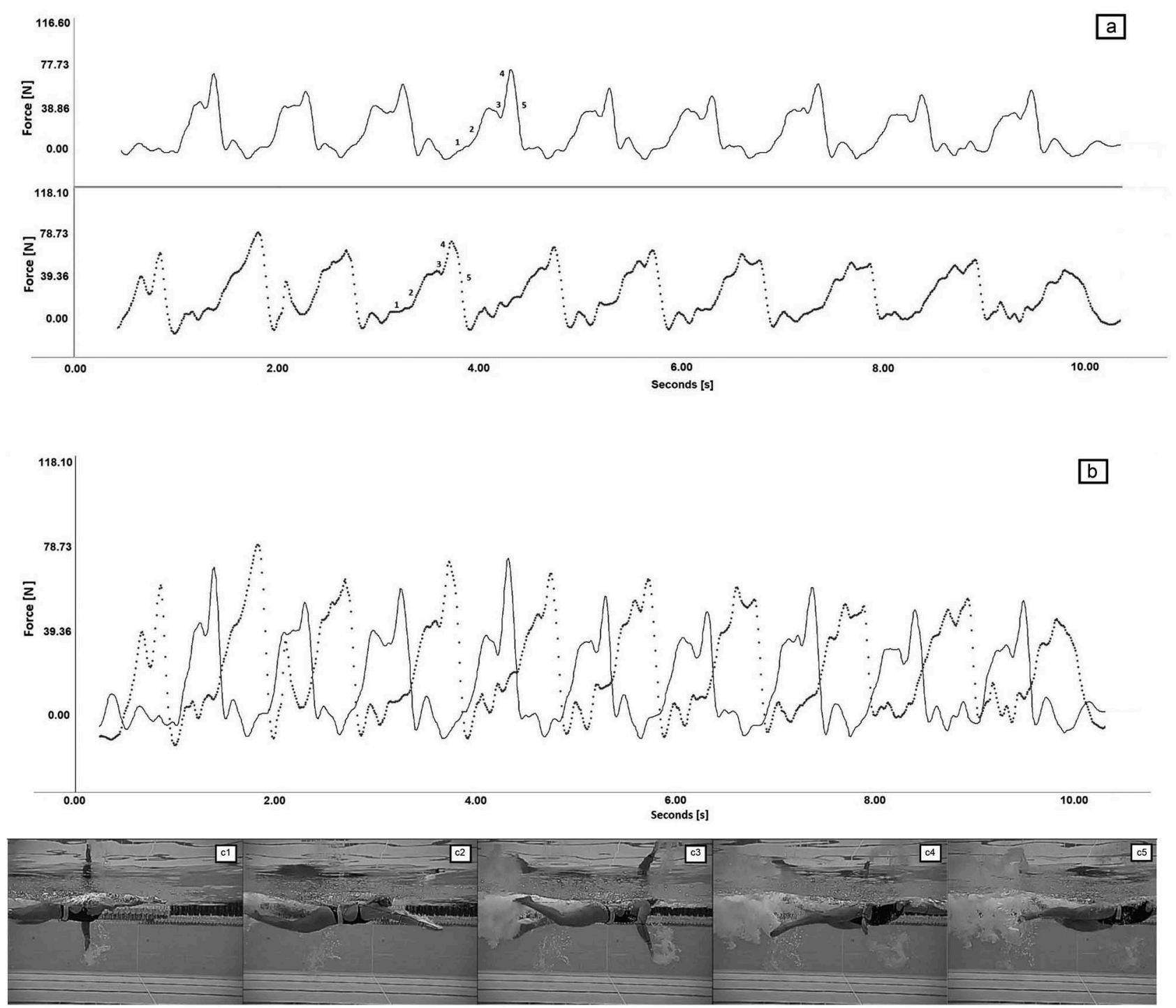

Figure 1. Panel (a) presents the thrust time-series for only the dominant (dash-line) and only the non-dominant (solid-line) upper-limbs. Panel (b) present the thrust time-series with both upper-limbs synchronized. 1 - hand's entry; 2 - downsweep; 3 - insweep; 4 - upsweep; 5 - exit and recovery. Panels (c) present an example of the right upper-limb stroke (underwater phase): C1 - hand's entry; C2 - downsweep; C3 - insweep; C4 - upsweep; C5 - exit and recovery.

significant inter-limb effect in the anthropometrics and thrust. The symmetry index indicated a symmetry between upper-limbs, since all variables were within the cutoff values previously indicated in Equation (2) (Table 2). Nonetheless, the $\mathrm{dF}$ showed the highest symmetry index (dF_dominant versus $\mathrm{dF}_{-}$non-dominant) with large effect size $(\mathrm{SI}=-5.57$; $d=0.51 ; p=0.135$ ) (Table 2).

Figure 1 depicts the thrust time-series for the dominant and non-dominant limbs, and full stroke. It is possible to note that the highest peak is achieved in the upsweep phase, and the lowest in the hand's entry/beginning of the downsweep (Figure 1). Overall, the dominant upper-limb did show higher (but not significant) mean (dominant: $37.88 \pm 6.61 \mathrm{~N}$; nondominant: $36.18 \pm 6.42 \mathrm{~N}$ ), and peak (dominant: $64.63 \pm 8.19$ $\mathrm{N}$; non-dominant: $64.49 \pm 10.69 \mathrm{~N}$ ) values of thrust (Tables 1 and 2). On the other hand, the $\mathrm{dF}$ was higher in the nondominant side (dominant: $50.24 \pm 5.14 \%$; non-dominant: 53.29 $\pm 6.93 \%$ ) (Table 1, Figure 1).
Prediction models are presented in Table 3. For both dominant and non-dominant side, the model retained as main predictors the respective HSA. For the dominant upper-limb, an increase in one unit $\left(\mathrm{cm}^{2}\right)$ by the dominant HSA led to a $0.293 \mathrm{~N}$ (95Cl: $0.117 ; 0.469 ; \mathrm{p}=0.005)$ increase in thrust. For the non-dominant HSA, an increase in one unit $\left(\mathrm{cm}^{2}\right)$ led to a $0.295 \mathrm{~N} \mathrm{(95Cl:} 0.063 ; 0.526 ; p=0.025)$ increase in thrust (Table 3). As for the full stroke cycle, the model retained the upper-body dry-land strength as main predictor. One unit $(\mathrm{kg})$ increased by the upper-body dry-land strength, led to a 0.397 $\mathrm{N}(95 \mathrm{Cl}: 0.189 ; 0.605 ; \mathrm{p}=0.002)$ increase in thrust (Table 3 ).

\section{Discussion}

This study aimed to assess anthropometric and thrust interlimb asymmetry, and determine the contribution of anthropometrics, and dry-land upper-body strength and power to the thrust of talented adolescent swimmers. The main results 
Table 1. Descriptive statistics and $95 \%$ confidence interval $(95 \mathrm{Cl})$ for all variables assessed.

\begin{tabular}{|c|c|c|c|c|}
\hline & Mean $\pm 1 S D$ & $95 \mathrm{Cl}$ & Minimum & Maximum \\
\hline Body mass $[\mathrm{kg}]$ & $66.61 \pm 7.76$ & $(62.62 ; 70.60)$ & 50.50 & 78.00 \\
\hline Height $[\mathrm{m}]$ & $1.73 \pm 0.08$ & $(1.69 ; 1.78)$ & 1.53 & 1.85 \\
\hline Arm span [m] & $1.79 \pm 0.11$ & $(1.73 ; 1.84)$ & 1.59 & 1.97 \\
\hline Dominant arm $[\mathrm{cm}]$ & $32.39 \pm 3.16$ & $(30.81 ; 33.96)$ & 27.00 & 40.50 \\
\hline Dominant forearm [cm] & $28.25 \pm 2.19$ & $(23.95 ; 32.54)$ & 23.60 & 31.90 \\
\hline Dominant upper-limb $[\mathrm{cm}]$ & $78.97 \pm 5.88$ & $(76.04 ; 81.90)$ & 69.00 & 91.00 \\
\hline Dominant hand surface area $\left[\mathrm{cm}^{2}\right]$ & $134.66 \pm 13.80$ & $(127.56 ; 141.75)$ & 104.83 & 153.44 \\
\hline Non-dominant arm $[\mathrm{cm}]$ & $32.11 \pm 3.19$ & $(30.52 ; 33.70)$ & 26.50 & 40.50 \\
\hline Non-dominant forearm $[\mathrm{cm}]$ & $28.02 \pm 1.92$ & $(24.25 ; 31.78)$ & 24.00 & 31.00 \\
\hline Non-dominant upper-limb [cm] & $78.50 \pm 5.77$ & $(75.63 ; 81.37)$ & 67.50 & 91.00 \\
\hline Non-dominant hand surface area $\left[\mathrm{cm}^{2}\right]$ & $136.75 \pm 15.58$ & $(128.73 ; 144.76)$ & 104.20 & 160.72 \\
\hline Dry-land upper-body strength $[\mathrm{kg}]$ & $55.58 \pm 14.31$ & $(48.22 ; 62.94)$ & 34.00 & 78.00 \\
\hline F_mean_dominant $[\mathrm{N}]$ & $37.88 \pm 6.61$ & $(34.48 ; 41.28)$ & 28.02 & 55.39 \\
\hline F peak dominant [N] & $64.63 \pm 8.19$ & $(60.41 ; 68.84)$ & 52.68 & 81.81 \\
\hline $\mathrm{dF}$ _dominant [\%] & $50.24 \pm 5.14$ & $(47.60 ; 52.89)$ & 37.15 & 60.37 \\
\hline $\mathrm{F}$ mean non-dominant $[\mathrm{N}]$ & $36.18+6.42$ & $(32.87: 39.48)$ & 26.56 & 52.64 \\
\hline F_peak non-dominant $[\mathrm{N}]$ & $64.49 \pm 10.69$ & $(58.99 ; 69.99)$ & 48.26 & 91.68 \\
\hline $\mathrm{dF}$ _non-dominant [\%] & $53.29 \pm 6.93$ & $(49.73 ; 56.86)$ & 38.18 & 65.82 \\
\hline F stroke cycle [N] & $74.06 \pm 11.91$ & $(67.93 ; 80.18)$ & 55.41 & 108.02 \\
\hline F_peak_stroke cycle [N] & $129.13 \pm 16.50$ & $(120.64 ; 137.61)$ & 102.09 & 173.49 \\
\hline $\mathrm{dF}$ stroke cycle [\%] & $51.71 \pm 4.87$ & (49.28:54.13) & 41.22 & 63.10 \\
\hline
\end{tabular}

F_mean_dominant - mean thrust of the dominant upper-limb; F_peak_dominant - peak thrust of the dominant upper-limb; dF_dominant - intracyclic variation of the dominant upper-limb force; $F$ _mean_non-dominant - mean thrust of the non-dominant upper-limb; F_peak_non-dominant peak thrust of the non-dominant upper-limb; $d F$ _non-dominant - intra-cyclic variation of the non-dominant upper-limb force; $F_{-}$stroke cycle -

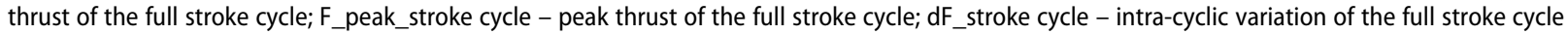
force.

Table 2. Inter-limb effect and symmetry index between dominant and non-dominant variables.

\begin{tabular}{|c|c|c|c|c|}
\hline Dominant vs non-dominant & $\mathrm{SI}(95 \mathrm{Cl})$ & d & F-ratio $(p)$ & Mean difference $(95 \mathrm{Cl})$ \\
\hline Arm $[\mathrm{cm}]$ & $0.88(-3.71 ; 5.47)$ & 0.09 & $0.069(0.795)$ & $0.277(-0.076 ; 0.631)$ \\
\hline Forearm $[\mathrm{cm}]$ & $0.75(-2.89 ; 4.39)$ & 0.11 & $0.112(0.740)$ & $0.230(-0.027 ; 0.487)$ \\
\hline Upper-limb [cm] & $0.59(-1.84 ; 3.03)$ & 0.08 & $0.059(0.809)$ & $0.472(-0.014 ; 0.958)$ \\
\hline Hand surface area $\left[\mathrm{cm}^{2}\right]$ & $-1.23(-8.48 ; 6.03)$ & 0.13 & $0.145(0.706)$ & $1.82(-0.72 ; 4.36)$ \\
\hline F_mean $[\mathrm{N}]$ & $3.94(-23.48 ; 31.37)$ & 0.23 & $0.465(0.500)$ & $1.44(-1.17 ; 4.05)$ \\
\hline F_peak [N] & $0.05(-28.54 ; 28.63)$ & 0.03 & $0.006(0.938)$ & $0.24(-4.91 ; 4.42)$ \\
\hline $\mathrm{dF}[\%]$ & $-5.57(-32.34 ; 21.20)$ & 0.51 & $2.346(0.135)$ & $3.03(-0.33 ; 6.39)$ \\
\hline
\end{tabular}

SI - symmetry index (\%); $95 \mathrm{Cl}$ - 95\% confidence interval; $d$ - Cohens d (effect size index); F-ratio - one-way ANOVA value; $p$ - ANOVA's significance value; Mean difference - mean difference in S.I. units; F_mean - mean thrust; F_peak - peak thrust; dF - intra-cyclic variation of the thrust.

Table 3. Hierarchical linear models' coefficients with $95 \%$ confidence interval $(95 \mathrm{Cl})$ are presented for all models computed.

\begin{tabular}{|c|c|c|c|}
\hline \multicolumn{4}{|l|}{ HLM models } \\
\hline Parameter fixed effect & Estimate (SE) & $95 \mathrm{Cl}$ & $\mathrm{p}$ \\
\hline \multicolumn{4}{|l|}{ F_mean_dominant } \\
\hline intercept & $37.718(1.172)$ & $35.421 ; 40.015$ & $<0.001$ \\
\hline Dominant hand surface area & $0.293(0.090)$ & $0.117 ; 0.469$ & 0.005 \\
\hline \multicolumn{4}{|l|}{ F_mean_non-dominant } \\
\hline intercept & $35.528(9.020)$ & $17.848 ; 53.207$ & 0.001 \\
\hline Non-dominant hand surface area & $0.295(0.118)$ & $0.063 ; 0.526$ & 0.025 \\
\hline \multicolumn{4}{|l|}{ F_stroke cycle } \\
\hline intercept & $51.748(5.603)$ & $40.766 ; 62.730$ & $<0.001$ \\
\hline Dry-land upper-body strength & $0.397(0.106)$ & $0.189 ; 0.605$ & 0.002 \\
\hline
\end{tabular}

showed a non-significant inter-limb effect for all anthropometric and thrust variables assessed. The thrust of the dominant and non-dominant limbs was Orelated to the correspondent HSA. On the other hand, the full stroke thrust was related to the upper-body strength and power.

Scientific knowledge has been reporting for several decades the importance of swimmer's body lengths (i.e. anthropometric features) to swimming performance $[1,36]$. It has been reported that faster swimmers are taller and bigger (i.e. present higher body lengths, widths and areas) in all age-groups from prepubescent to adult [36,37]. Nonetheless, anthropometric symmetries/asymmetries and their relationship with thrust are less explored by the swimming community. Our data showed a non-significant inter-limb effect for the anthropometric features (Table 2). Nevertheless, all dominant lengths were higher (but not significantly) in comparison to non-dominant ones (Table 1). Similarly to our results, the literature reported bilateral asymmetries regarding human's upper-limbs favouring the 
dominant side [38,39]. Curiously, the non-dominant upper-limb showed a higher HSA. Nonetheless, hand asymmetry related to limb dominance is reported in the literature [40]. It is common for subjects to present higher hand lengths and widths in the non-dominant upper-limb [40].

The literature suggested a direct relationship between behavioural and morphological asymmetry through mechanically driven bone growth and remodeling [39]. Bilateral asymmetries in movement patterns may originate from genetic and early environmental factors [9]. Indeed, increased asymmetries were verified between the playing (dominant) and nonplaying (non-dominant) arms of sport athletes where the upper-limbs are mostly used [41]. However, in the particular case of swimming, both upper-limbs are used to produce thrust. So, based on the environmental perspective of the bilateral asymmetry, one might claim that swimmers could also present such asymmetries. Present data showed that differences were noted between dominant and nondominant upper-limb thrust (higher in the dominant upperlimb), but without a meaningful and significant inter-limb effect (Tables 1 and 2, Figure 1). Here, the symmetry index revealed a symmetry between upper-limbs, since it was within the cutoff limits $(-10 \%<\mathrm{SI}<10 \%)$. One study showed interlimb asymmetries in force production (tethered swimming) in high-level swimmers (in similar age-group) in the front crawl [20]. The authors also compared fastest versus slowest swimmers' performances, and concluded that the inter-limbs asymmetries verified within groups (but not between groups) did not affect negatively the performance [20].

Others reported that the fastest swimmers exhibit lower peak and mean force asymmetries (tethered swimming) in comparison to the slowest swimmers, suggesting that this may be a handicap to enhancing swimming performance [7]. Indeed, it was indicated that asymmetries in swimming may limit swimming performance by reducing the capacity to produce thrust [9,32]. Moreover, such controversial outcomes were observed in the tethered-swimming method. It was suggested that the forces generated by a tethered swimmer are considerably different from those generated while swimming 'freely' due to the different velocity of the swimmer relative to the water [9]. Moreover, the hips (point of the body where the tether is attached) do not represent the effect of the propulsive actions on the acceleration of the center of mass [9]. Thus, it can be suggested that more research is needed to understand this phenomenon in swimming.

Despite studies having analyzed thrust and inter-limb asymmetries $[7,10]$, less is known about how thrust can be determined. It could be suggested that anthropometric features and upper-body dry-land strength and power could be related/could contribute to front crawl thrust. Hierarchical linear modeling retained as main predictors of the dominant and non-dominant limbs the corresponding HSA, where a positive relationship was observed (Table 3). Propulsive force presents a direct and positive relationship to the surface area [1]. That is, an increase in the surface area will lead to an increase in the propulsive drag (i.e. thrust) [19]. Both experimental [42] and numerical studies [43]appear to indicate that an increase in the hand's area led to an increase in the propulsive force. An increase of the drag of the hand by spreading fingers reduces the slip velocity between the hand and the water, diminishing the power dissipated for propulsion [14]. Thus, it might be suggested that swimmers should 'increase' their hands' surface area by spreading their fingers. It was noted that the hand surface area 'enlarged' with a small distance between fingers $(0.32 \mathrm{~cm})$ increased the projection surface area of the hand, and hence the force production [44]. It seems that in a turbulent flow (like in an arm-pull) some kind of barrier between the fingers may be formed, leading to an 'increase' in the hand's surface area [44]. Nonetheless, this spread should be maximized but not greatly enlarged. Contrarily, a higher finger spread $(0.64 \mathrm{~cm})$ allowed the water to flow freely, and consequently did not increase force production [44]. Despite the fact that the upper-limb (as an articulated segment) may be determinant for propelling efficiency [16], the hand plays a key-role in front crawl thrust [15].

For the full stroke cycle, the model retained the upper-body strength and power variable (Table 3 ). In order to produce thrust, swimmers should be able to move water backwards (both upper-limbs) with as much force as possible. In this sense, it could be speculated that a direct and positive relationship between the force that a swimmer can produce on dry-land and in-water should occur. Indeed, such a relationship between dry-land strength and swimming performance has been suggested in similar age-groups of swimmers (age and competitive level) $[8,23]$. However, less is known about such a relationship between dry-land and in-water force production (i.e. thrust). Dry-land strength and power training have been used by the swimming community as an in-water training complement. Aspenes et al. [23] reported that after a dry-land strength training, swimmers significantly increased their dry-land strength output, as well as their in-water force production. Moreover, it was shown that upper-limbs' dry-land strength measured on a bench press presented a high correlation with in-water force production [8]. Thus, it might be suggested that a direct and positive effect between dry-land strength and in-water force production (i.e. thrust) exists $[8,23]$.

Talented adolescent swimmers exhibit a non-significant inter-limb asymmetry (i.e. symmetry) in anthropometric and thrust variables. Nonetheless, the dominant upper limb presented higher lengths (but lower HSA). The same trend was verified for the thrust. The dominant upper-limb presented a higher thrust (but non-significant), revealing a symmetry. Indeed, in the front crawl, both upper-limbs are responsible for the majority of the total thrust $[12,16]$. Nevertheless, others did show inter-limb asymmetries in a similar age-group of swimmers [20]. It might be argued that such inter-limb asymmetries indicate that swimmers use the dominant upper-limb to produce higher thrust, and the non-dominant for balance [21]. However, and accepting this rationality, it could be pointed out that such a phenomenon may occur even without inter-limb asymmetries (as the present data showed). Indeed, swimmers' dominant upper-limb presented higher values of thrust, despite a lower HSA (when comparing to the nondominant). This could be due to: (i) the dominant upper-limb 
only could have higher indexes of dry-land strength than the non-dominant limb [45], and; (ii) despite presenting lower HSA, the dominant upper-limb may overcome this factor with a higher motor control of that specific upper-limb. This may lead to a higher efficient use of force [21].

Nonetheless, each HSA (anthropometric) was responsible for the corresponding upper-limb thrust. The upper-body dry-land strength and power variables were responsible for the full stroke cycle thrust. Consequently, coaches and practitioners should aim to minimize notable differences (significant or not) between upper limbs, as this may negatively affect the full stroke cycle thrust, and potentially the performance [9]. Coaches should be aware that 'maximizing' the swimmer's HSA with a small finger spread, and increasing their upper-body strength and power could lead to a higher thrust, and hence to a performance enhancement.

The main limitations of this study can be considered to be as follows: (i) other upper-body anthropometric variables could be included (such as upper-limb girths and chest perimeters), and; (ii) individual upper-limbs' strength (i.e. dominant and nondominant) could give deeper insights. Additionally, it is suggested to measure the force production in each arm-pull phase (i.e. downsweep, insweep, and upsweep), and relate it to the pitch and sweepback angles performed.

\section{Conclusion/summary}

Talented adolescent swimmers exhibit a non-significant interlimb asymmetry in anthropometric and thrust variables. Each HSA (anthropometric) was responsible for the corresponding upper-limb thrust (i.e. dominant and non-dominant). The upperbody dry-land strength and power variable were responsible for the full stroke cycle thrust. Coaches should be aware that maximizing the swimmer's HSA through a small finger spread, and increasing their upper-body strength and power could lead to a higher thrust, and possibly to a performance enhancement.

\section{Acknowledgments}

This project was supported by the National Funds through FCT-Portuguese Foundation for Science and Technology (UID/DTP/04045/2019), and the European Fund for regional development (FEDER) allocated by the European Union through the COMPETE 2020 Programme (POCI-01-0145FEDER-006969). We would like also to thank the support of the University of Beira Interior and Santander Universities (Bolsa BIPD/ICIFCSH-Santander Universidades-UBI/2017).

\section{Declaration of interest}

The authors have no conflict of interest.

\section{ORCID}

Jorge E Morais (D) http://orcid.org/0000-0002-6885-0648 Mário C Marques (DD http://orcid.org/0000-0002-5812-1580 Tiago M Barbosa (D) http://orcid.org/0000-0001-7071-2116

\section{References}

1. Toussaint HM, Beek PJ. Biomechanics of competitive front crawl swimming. Sports Med. 1992;13(1):8-24.

2. Barbosa TM, Morais JE, Marques MC, et al. Hydrodynamic profile of young swimmers: changes over a competitive season. Scand J Med Sci Sports. 2015;25(2):184-196.

3. van Houwelingen J, Schreven S, Smeets JB, et al. Effective propulsion in swimming: grasping the hydrodynamics of hand and arm movements. J Appl Biomech. 2017;33(1):87-100.

4. Gatta G, Cortesi M, Zamparo P. The relationship between power generated by thrust and power to overcome drag in elite short distance swimmers. PloS One. 2016;11(9):e0162387.

5. Matsuuchi K, Miwa T, Nomura T, et al. Unsteady flow field around a human hand and propulsive force in swimming. J Biomech. 2009;42:42-47.

6. Cappaert JM, Pease DL, Troup JP. Three-dimensional analysis of the men's 100m freestyle during the 1992 olympic games. J Appl Biomech. 1995;11:103-112.

7. Dos Santos KB, Pereira G, Papoti M, et al. Propulsive force asymmetry during tethered-swimming. Int J Sports Med. 2013;34:606-611.

8. Morouço P, Neiva N, González-Badillo JJ, et al. Associations between dry land strength and power measurements with swimming performance in elite athletes: a pilot study. J Hum Kinet. 2011;29:105-112.

9. Sanders R, Thow J, Alcock A, et al. How can asymmetries in swimming be identified and measured? J Swim Res. 2012;19.

10. Pereira GS, Schutz GR, Ruschel C, et al. Propulsive force symmetry generated during butterfly swimming. Rev Bras Cineantropom Desempenho Hum. 2015;17(6):704-712.

11. Havriluk R. Validation of a criterion measure for swimming technique. J Swim Res. 1988;4(4):11-16.

12. Ribeiro J, Figueiredo $P$, Sousa $A$, et al. $V_{2}$ kinetics and metabolic contributions during full and upper body extreme swimming intensity. Eur J Appl Physiol. 2015;115(5):1117-1124.

13. Guinard B, Rouard A, Chollet D, et al. Behavioral dynamics in swimming: the appropriate use of inertial measurement units. Frontiers Psychol. 2017;8:383.

14. van Houwelingen J, Willemsen $D$, Kunnen $R$, et al. The effect of finger spreading on drag of the hand in human swimming. J Biomech. 2017;63:67-73.

15. Bilinauskaite $M$, Mantha VR, Rouboa Al, et al. Computational fluid dynamics study of swimmer's hand velocity, orientation, and shape: contributions to hydrodynamics. Biomed Res Int. 2013;2013: ID140487.

16. Zamparo P. Effects of age and gender on the propelling efficiency of the arm stroke. Eur J Appl Physiol. 2006;97:52-58.

17. Zamparo $P$, Pendergast DR, Mollendorf J, et al. An energy balance of front crawl. Eur J Appl Physiol. 2005;94:134-144.

18. Sidelnik NO, Young BW. Optimising the freestyle swimming stroke: the effect of finger spread. Sports Eng. 2006;9:129-135.

19. Kjendlie PL, Stallman R. Morphology and swimming performance. In: Seifert L, Chollet D, Mujika I, editors. World book of swimming: from science to performance. New York (NY): Nova Science Publishers; 2011. p. 203-222.

20. Morouço PG, Marinho DA, Fernandes RJ, et al. Quantification of upper limb kinetic asymmetries in front crawl swimming. Hum Mov Sci. 2015;40:185-192.

21. Seifert L, Chollet D, Allard P. Arm coordination symmetry and breathing effect in front crawl. Hum Mov Sci. 2005;24:234-256.

22. Garrido N, Marinho DA, Reis VM, et al. Does combined dry land strength and aerobic training inhibit performance of young competitive swimmers? J Sports Sci Med. 2010;9:300-310.

23. Aspenes S, Kjendlie PL, Hoff J, et al. Combined strength and endurance training in competitive swimmers. J Sports Sci Med. 2009;8:357-365.

24. Weston $M$, Hibbs $A E$, Thompson $K G$, et al. Isolated core training improves sprint performance in national-level junior swimmers. Int J Sports Physiol Perform. 2015;10:204-210. 
25. Nasirzade A, Ehsanbakhsh A, llbeygi S, et al. Relationship between sprint performance of front crawl swimming and muscle fascicle length in young swimmers. J Sports Sci Med. 2014;13:550-556.

26. Sanchez-Medina L, Perez CE, Gonzalez-Badillo JJ. Importance of the propulsive phase in strength assessment. Int J Sports Med. 2010;31:123-129.

27. Morais JE, Jesus S, Lopes $V$, et al. Linking selected kinematic, anthropometric and hydrodynamic variables to young swimmer performance. Pediatr Exerc Sci. 2012;24(4):649-664.

28. Wang R, Hoffman JR, Sadres $E$, et al. Evaluating upper-body strength and power from a single test: the ballistic push-up. J Strength Cond Res. 2017;31(5):1338-1345.

29. Pérez-Olea Jl, Valenzuela PL, Aponte $C$, et al. Relationship between dryland strength and swimming performance: pull-up mechanics as a predictor of swimming speed. J Strength Cond Res. 2018;32:1637-1642.

30. Loturco I, Kobal R, Moraes JE, et al. Predicting the maximum dynamic strength in bench press: the high precision of the bar velocity approach. J Strength Cond Res. 2017;31(4):1127-1131.

31. Morouço PG, Barbosa TM, Arellano R, et al. Intracyclic variation of force and swimming performance. Int J Sports Physiol Perform. 2018;13(7):897-902.

32. Tourny-Chollet C, Seifert L, Chollet D. Effect of force symmetry on coordination in crawl. Int J Sports Med. 2009;30:182-187.

33. Diedenhofen B, Musch J. cocor: a comprehensive solution for the statistical comparison of correlations. PLoS One. 2015;10(4):e0121945.

34. Cohen J. Statistical power analysis for the behavioral sciences. 2nd ed. Hillsdale, NJ: Lawrence Earlbaum Associates; 1988.

35. Raudenbush S, Bryk A, Cheong YF, et al. HLM7 Hierarchical linear and nonlinear modeling user manual: user guide for scientific software international's (S.S.I.) program. Lincolnwood, IL: Scientific Software International; 2011.

36. Nevill AM, Oxford SW, Duncan MJ. Optimal body size and limb length ratios associated with $100 \mathrm{~m}$ personal-best swim speeds. Med Sci Sports Exerc. 2015;47(8):1714-1718.

37. Morais JE, Silva AJ, Marinho DA, et al. Determinant factors of long-term performance development in young swimmers. Int J Sports Physiol Perform. 2017;12(2):198-205.

38. Auerbach BM, Ruff CB. Limb bone bilateral asymmetry: variability and commonality among modern humans. J Hum Evol. 2006;50:203-218.

39. Steele J. Handedness in past human populations: skeletal markers. Laterality. 2000;5:193-220.

40. Barut C, Sevinc O, Sumbuloglu V. Evaluation of hand asymmetry in relation to hand preference. Coll Antropol. 2011;35(4):1119-1124.

41. Bass SL, Saxon L, Daly RM, et al. The effect of mechanical loading on the size and shape of bone in pre-, peri-, and post-pubertal girls: a study in tennis players. J Bone Miner Res. 2002;7:2274-2280.

42. Toussaint HM, Janssen T, Kluft M. Effect of propelling surface size on the mechanics and energetics of front crawl swimming. J Biomech. 1991;24:205-211.

43. Vilas-Boas JP, Ramos RJ, Fernandes RJ, et al. Hydrodynamic analysis of different finger positions in swimming: a computational fluid dynamics approach. J Appl Biomech. 2015;31(1):48-55.

44. Marinho DA, Barbosa TM, Reis VM, et al. Swimming propulsion forces are enhanced by a small finger spread. J Appl Biomech. 2010;26:87-92.

45. Batalha N, Dias S, Marinho DA, et al. The effectiveness of land and water based resistance training on shoulder rotator cuff strength and balance of youth swimmers. J Hum Kinet. 2018;62:91-102. 